\title{
A HARD NUT TO CRACK: SUCCESSFUL RESOLUTION OF SOMATIC DELUSION WITH ELECTROCONVULSIVE THERAPY IN A CASE OF LATE ONSET PERSISTENT DELUSIONAL DISORDER
}

\author{
Rajeev Ranjan ${ }^{1}$, Santanu Nath ${ }^{2}$, Gabby Sethi ${ }^{1}$ \& Pankaj Kumar ${ }^{1}$ \\ ${ }^{1}$ Department of Psychiatry, All India Institute of Medical Sciences (AIIMS), Patna, Bihar, India \\ ${ }^{2}$ Department of Psychiatry, All India Institute of Medical Sciences (AIIMS), Deoghar, Jharkhand, India
}

received: 22.2.2021;

revised: 15.3.2021;

accepted: 6.4 .2021

\section{INTRODUCTION}

A delusion concerning a somatic theme is entertained when a sufferer holds a fixed, firm and unshakeable belief that $\mathrm{s} /$ he has an illness related to body structure or function. Often such a delusion (somatic/ hypochondriacal delusion) becomes resistant to usual psychiatric treatment and it lingers for years causing significant psychosocial dysfunction (Skelton et al. 2015). We report a case of an old lady (informed consent obtained), who harboured a somatic delusion that was not amenable to change with usual psychotropics but which responded dramatically with Electroconvulsive Therapy (ECT) while trying to find a neurobiological explanation for the same.

\section{CASE PRESENTATION}

A 55-year-old Hindu married lady with family history of late onset psychosis in mother, presented with an insidious onset and a continuous deteriorating course of 3 years duration of complains of multiple somatic symptoms involving her throat, tooth and facial region. She stopped doing her household chores and used to remain preoccupied with her thought. Before the current presentation, she visited many physicians and underwent multiple investigations but no proper explanation of her symptoms could be found. On Mental status Examination, she had an anxious and restricted affect with thought content revealing a belief that her tongue and food pipe had narrowed which hindered her ability to swallow both solid and liquid food. She would tell that if taken, the food will not reach her stomach. She held this thought with conviction and refused to accept any evidence to the contrary thus revealing somatic delusion. She however had no perceptual disturbances. She was provisionally diagnosed as persistent delusional disorder; somatic type and was admitted. She was evaluated to have normal routine and biochemical blood parameters (complete haemogram, renal, liver and thyroid function tests) and her Magnetic Resonance Imaging Scan of brain was unremarkable. She was managed with subsequent adequate trials of Risperidone (upto 8 $\mathrm{mg}$ /day) and Olanzapine (upto $15 \mathrm{mg} /$ day) which lead to no response. She held her somatic delusion with the same conviction as earlier. She subsequently developed depressive symptoms for which she was started on Venlafaxine upto $150 \mathrm{mg} /$ day. Considering no response in her thought content (somatic delusion), a course of modified bi-temporal ECT was started with proper consent. After the $5^{\text {th }} \mathrm{ECT}$, she started to respond which was evident in gradual resolution of her delusion. She was administered a total of 8 modified ECTs and she responded significantly with substantial reduction of the conviction of her delusion. She was discharged on Olanzapine $15 \mathrm{mg}$ /day and on subsequent follow ups she was maintaining well.

\section{DISCUSSION}

Persistent delusional disorder; somatic type is also known as Monosymptomatic Hypochondriacal Psychosis (MHP) in which a single delusion involving somatic structure and/or function is held with conviction in absence of any other symptoms and without gross personality and functional deterioration (Munro 1988). Such patients often visit dermatologists, plastic surgeons and general surgeons and they end up in a never-ending trail of investigations and treatments (including surgeries too) but ultimately no satisfactory response. Various antipsychotics, antidepressants, mood stabilizers and non-pharmacological therapies have been advocated for its treatment but overall, they are difficult to treat (Skelton et al. 2015).

Till date there are sparse data on ECT as an effective treatment option for somatic symptoms and more so when it takes a delusional form. There are literatures which suggest a role of ECT in somatic/hypochondriacal delusions (Table 1) (Ota et al. 2003, Newmark \& Al-Samarrai 2004. Uezato et al. 2012, Dols et al. 2012, Wong 2017, Cohen et al. 2019). In all the cases described, the patients were elderly who harboured a somatic/ hypochondriacal delusion on a backdrop of a diagnosis of either depressive disorder or a primary psychotic illness (schizoaffective disorder, delusional disorder) and which were all resistant to pharmacological approaches, 
Table 1. ECT treatment in somatic/hypochondriacal delusion

\begin{tabular}{llc}
\hline Author & \multicolumn{1}{c}{ Clinical profile } & ECT sessions and response \\
\hline Ota et al. 2003 & $\begin{array}{l}\text { A 72-year-old male having somatic delusion invol- He responded completely after } 6 \text { modified } \\
\text { ving his jaw, mouth and oesophagus. He was diag- ECTs which paralleled with improvement of }\end{array}$ \\
& $\begin{array}{l}\text { nosed as delusional disorder; somatic type and had regional cerebral blood flow in the left tem- } \\
\text { past depressive episodes. }\end{array}$ & poral and parietal lobes shown by SPECT.
\end{tabular}

Newmark \& Al- 49-year-old lady having a 3 years history of hypo- 4 sessions of ECT resulted in substantial Samarrai 2004 chondriacal concern of many diseases including cancer improvement in reducing her somatic along with secondary depression and anxiety symp- preoccupation and subsequent functional toms. She was treated with antidepressants, antianxiety improvement. drugs and antipsychotics without success.

Uezato et al. 2012 A 53-year-old lady having a history of mild depression 10 sessions of bilateral modified ECT was presented with somatic delusion involving the oral administered which resulted in significant area (oral cenesthopathy). She had failed trials with improvement. This also resulted in a demonmultiple antidepressants and antipsychotics. strable change in SPECT scan of her brain.

Dols et al. 2012 A 72-year-old lady having hypochondriacal delusion 13 sessions of unilateral modified ECT involving her abdomen which had a poor response to administered resulting in complete symppsychotropics. tom remission that lasted for 2.5 years.

Wong et al. 2017 A 67-year-old male with psychotic depression had A course of 6 bilateral modified ECT was hypochondriacal delusion involving his oesophagus given which resulted in substantial improand abdomen. He had failed trials of antidepressants vement in hypochondriacal delusion. and antipsychotics.

Cohen et al. 2019 A 48-year-old lady diagnosed as schizoaffective A session of 6 bitemporal modified ECTs disorder had a somatic delusion involving abdomen, resulted in substantial resolution of her jaw and the genitourinary system which was resistant somatic delusion. to even clozapine.

but responded dramatically with modified ECT that resulted in significant functional improvement. These points to two important conclusions, firstly, somatic/ hypochondriacal delusions which are one of the most refractory to conventional pharmacological treatment can be amenable to change with somatic treatments like ECT and secondly, ECT is an important treatment option in elderly population where psychotropics can pose physical risks.

The mechanism by which ECT exerts a change in delusions (somatic delusion in our case) per se is dubious but can be hypothesized to be similar to its effect on the treatment for overall psychosis. There are propositions as to how ECT has an effect on somatoform disorders. A potential to produce a long-term structural change in the limbic system and in the prefrontal cortex has been proposed along with findings from several studies that demonstrated its role in altering regional cerebral blood flows and metabolic rates (Yatham et al. 2000, Nobler et al. 2001, Dukart et al. 2014). Whether these mechanisms also work for its effect specifically on mitigating somatic delusion is unclear.

Nevertheless, this case emphasizes that ECT can always be considered as an important treatment option when encountering a case of resistant somatic delusion even in elderly when other conventional pharmacological options are exhausted. Longitudinal studies are needed that will focus on long term outcome in this group of patients.

\section{Acknowledgements: None.}

\section{Conflict of interest: None to declare.}

\section{Contribution of individual authors:}

Rajeev Ranjan, Gabby Sethi \& Pankaj Kumar saw the case in question and managed the case.

Santanu Nath provided expert opinion on the further management of this case.

The initial draft of the manuscript was written by Santanu Nath \& Rajeev Ranjan while it was provided with intellectual inputs by Gabby Sethi \& Pankaj Kumar.

Relevant literature search was done by Santanu Nath.

The full manuscript was reviewed by all the authors before submission.

\section{References}

1. Cohen JL, Vu MT, Beg MA, et al. Successful Resolution of Prominent Somatic Delusions Following Bi-temporal Electroconvulsive Therapy in a patient with TreatmentResistant Schizoaffective Disorder. Psychopharmacol Bull 2019; 49:52-56

2. Dols A, Rhebergen D, Eikelenboom P, et al. Hypochondriacal delusion in an elderly woman recovers quickly with electroconvulsive therapy. Clin Pract. 2012; 2:e11

3. Dukart, J., Regen, F., Kherif, F., et al. Electroconvulsive therapy-induced brain plasticity determines therapeutic outcome in mood disorders. Proceedings of the National 
Academy of Sciences of the United States of America. 2014; 111, 1156-1161

4. Munro A. Monosymptomati chypochondriacal psychosis. Br J Psychiatry Suppl 1988; 2:37-40

5. Newmark TS, Al-Samarrai S. Hypochondriasis and ECT. Psychosomatics. 2004;45:90-91

6. Nobler MS, Oquendo MA, Kegeles LS, et al. Decreased regional brain metabolism after ect. Am J Psychiatry. 2001; 158:305-8

7. Ota M, Mizukami K, Katano T, et al. A case of delusional disorder, somatic type with remarkable improvement of clinical symptoms and single photon emission computed tomograpy findings following modified electroconvulsive therapy. Prog Neuropsychopharmacol Biol Psychiatry. 2003;27:881-884
8. Skelton M, Khokhar WA, Thacker SP. Treatments for delusional disorder. Cochrane Database Syst Rev. 2015;5:CD009785

9. Uezato A, Yamamoto N, Kurumaji A, et al. Improvement of asymmetrical temporal blood flow in refractory oral somatic delusion after successful electroconvulsive therapy. J ECT. 2012;28:50-1

10. Wong M. Hypochondriacal delusion in an elderly man with good response to electroconvulsive therapy but complicated with febrile reaction. Archives of Depression and Anxiety 2017:038-41

11. Yatham LN, Clark CC, Zis AP. A preliminary study of the effects of electroconvulsive therapy on regional brain glucose metabolism in patients with major depression. $J$ ECT. 2000; 16:171-176

Correspondence:

Assistant Professor Santanu Nath, MBBS, MD, DNB

Department of Psychiatry, All India Institute of Medical Sciences (AIIMS)

Deoghar, PTI Campus, Daburgram, District: Deoghar, PIN: 814142, Jharkhand, India

E-mail: beta.santanu@gmail.com 